\title{
Clostridium sordellii genome analysis reveals plasmid localized toxin genes encoded within pathogenicity loci
}

\author{
Edward C. Couchman ${ }^{1}$, Hilary P. Browne ${ }^{2}$, Matt Dunn², Trevor D. Lawley², J. Glenn Songer ${ }^{3}$, Val Hall', Liljana Petrovska ${ }^{5}$,
} Callum Vidor ${ }^{6}$, Milena Awad ${ }^{6}$, Dena Lyras ${ }^{6}$ and Neil F. Fairweather ${ }^{1 *}$

\begin{abstract}
Background: Clostridium sordellii can cause severe infections in animals and humans, the latter associated with trauma, toxic shock and often-fatal gynaecological infections. Strains can produce two large clostridial cytotoxins (LCCs), TCsL and TCsH, related to those produced by Clostridium difficile, Clostridium novyi and Clostridium perfringens, but the genetic basis of toxin production remains uncharacterised.

Results: Phylogenetic analysis of the genome sequences of 44 strains isolated from human and animal infections in the UK, US and Australia placed the species into four clades. Although all strains originated from animal or clinical disease, only 5 strains contained LCC genes: 4 strains contain tcsL alone and one strain contains tcsL and tcsH. Four toxin-positive strains were found within one clade. Where present, tcs $L$ and $t c s H$ were localised in a pathogenicity locus, similar to but distinct from that present in C. difficile. In contrast to C. difficile, where the LCCs are chromosomally localised, the C. sordellii tcs $L$ and tcsH genes are localised on plasmids. Our data suggest gain and loss of entire toxigenic plasmids in addition to horizontal transfer of the pathogenicity locus. A high quality, annotated sequence of ATCC9714 reveals many putative virulence factors including neuraminidase, phospholipase $C$ and the cholesterol-dependent cytolysin sordellilysin that are highly conserved between all strains studied.

Conclusions: Genome analysis of $C$. sordellii reveals that the LCCs, the major virulence factors, are localised on plasmids. Many strains do not contain the LCC genes; it is probable that in several of these cases the plasmid has been lost upon laboratory subculture. Our data are consistent with LCCs being the primary virulence factors in the majority of infections, but LCC-negative strains may precipitate certain categories of infection. A high quality genome sequence reveals putative virulence factors whose role in virulence can be investigated.
\end{abstract}

Keywords: Clostridium sordellii, Large Clostridial Cytotoxin, Plasmid, PaLoc

\section{Background}

Clostridium sordellii is an anaerobic, Gram-positive, spore-forming species of bacterium [1]. It is commonly found in the soil, and can be found in the gut of animals, including humans [2]; it has also been identified in the vaginal microbiota of a small percentage of women [3]. Human C. sordellii infections are rare but can cause a variety of serious diseases, including myonecrosis and toxic shock syndrome [4-6]. Infections

\footnotetext{
* Correspondence: n.fairweather@imperial.ac.uk

${ }^{1}$ Department of Life Sciences, Centre for Molecular Bacteriology and

Infection, Imperial College London, London SW7 2AZ, UK

Full list of author information is available at the end of the article
}

are most often associated with traumatic injury, surgery, intravenous drug use and gynaecological events (childbirth, abortion and miscarriage). They generally occur in otherwise healthy individuals but have a high mortality rate; wound infections have a mortality rate of $\sim 50 \%$, while gynaecological infections are almost universally fatal [3]. An association between C. sordellii and the abortioninducing drug combination mifepristone-misoprostol has been noted; misoprostol may facilitate infection by suppressing the activity of the innate immune system [7]. Early symptoms of infection are non-specific, but within hours of presentation most patients develop hypotension and tachycardia that rapidly progresses to multi-organ 
failure [3]. C. sordellii is also an important animal pathogen, causing disease in farm animals including sheep, horses and cattle $[8,9]$.

The bacteria can produce several toxins and virulence factors, of which lethal toxin (TcsL) and haemorrhagic toxin $(\mathrm{TcsH})$ are considered the most potent [10]. TcsL and $\mathrm{TcsH}$ are members of the Large Clostridial Cytotoxin (LCC) family [11] and are closely related to C. difficile toxins $T c d B$ and $T c d A$ respectively [12]. Correlation of TcsL production and disease suggests that TcsL is required for C. sordellii to induce toxic shock syndrome [10]; indeed, while the $t c s L_{/}^{+} t c s H^{-}$strain ATCC9714 is rapidly lethal in a mouse model of infection, mutation of tcsL results in a complete loss of virulence [13]. Additionally, certain strains of $C$. sordellii lacking LCCs have been associated with less severe infections in humans $[14,15]$.

Other putative virulence factors produced by $C$. sordellii include neuraminidase (nanS), phospholipase C (PLC, csp) and, notably, the cholesterol-dependent cytolysin sordellilysin $(s d l)$ [16]. An earlier study of a collection of 14 strains isolated from human cadaver tissues revealed that only one strain encoded and produced TcsL, and while all strains encoded the $s d l$ gene, only five produced the protein, suggesting that not all strains are able to express their $s d l$ genes, at least under the conditions used [16]. This variation in toxin production between strains may well account for the variation in disease type and severity associated with this species.

We have undertaken a genomic analysis of a collection of C. sordellii strains derived mainly from cases of clinical or veterinary disease. The majority of strains do not encode $t c s L$ or $t c s H$, but all strains encode the putative virulence factors sordellilysin, phospholipase $\mathrm{C}$ and neuraminidase. Where tcsL or tcsH are present, they are localised on a plasmid. A high quality draft genome sequence of strain ATCC9714 reveals numerous other putative virulence factors and will allow future studies on the pathogenesis of $C$. sordellii infection.

\section{Results}

\section{C. sordellii strains divide into four clades}

A collection of 44 strains of $C$. sordellii was assembled from both clinical and veterinary cases of disease within the UK, USA and Australia (Table 1). Genomic DNA was extracted and sequenced and core gene sequences compared and analysed, generating a phylogenetic tree (Fig. 1). This shows the strains can be divided into 4 primary clades. Five strains appear to be outliers from our selection, not belonging to any clade (indicated with an * in Fig. 1), though it is possible that were more strains to be added these would fall into new, distinct clades. Interestingly, no clade is specific to any country-oforigin, or is specifically associated with either clinical or veterinary disease.

\section{The majority of $C$. sordellii strains do not encode the Large Clostridial Cytotoxins TcsL and TcsH}

When analysing the initial sequence assemblies we noticed that few strains appeared to contain the genes encoding the LCCs TcsL and TcsH. Five strains contain the tcsL gene (ATCC9714, JGS444, JGS445, JGS6382 and JGS6364), and of these only JGS6382 also contains $t c s H$. The four $t c s L^{+} / t_{c s} H^{-}$strains are closely related, all found in Clade 1 of our phylogenetic tree, and all contain identical 5 ' fragments of the $t c s H$ gene, as previously reported for ATCC9714 [17]. No strain contains $t c s H$ without tcsL. PCR using primers specific for $t c s L$ or $\mathrm{t} c s H$ confirmed the presence of the toxin genes only in these genomes (data not shown). Strains encoding $t c s L$, or $t c s L$ and $t c s H$, are indicated by $\mathbf{p L}$ or $\mathbf{p L H}$, respectively in Fig. 1 (and see below). Intriguingly, tcsL was absent from strain UMC164. UMC164 is part of clade 1 and therefore closely related to all four $t c s L^{+} / t c s H^{-}$strains, and a previous analysis in 2006 indicated that it, too, was $t c s L^{+} / t_{c s} H^{-}$[16]. UMC164 appears therefore to have lost the $t c s L$ gene upon culturing in the laboratory.

\section{The C. sordellii LCC genes are located on one of two plasmids found in ATCC9714}

After initial optical mapping of the ATCC9714 genome, it was determined that considerably more sequence remained unmapped than gaps were estimated to remain in the chromosome, suggesting that some of this sequence was localized on extra-chromosomal elements. A large contig of $103.8 \mathrm{~kb}$ of unmapped sequence was assembled which could not be optically mapped to the chromosome. PCR demonstrated the circular nature of this element, which we named plasmid pCS1-1. pCS1-1 contains $t c s L$, the alternative sigma factor $t c s R$ and the presumed holin protein $t c s E$, present in a pathogenicity locus (PaLoc) in the relative orientations described previously [17]. The PaLoc region of pCS1-1 and the surrounding genes are shown in Fig. 2. pCS1-1 also encodes several proteins likely required for plasmid replication: a replication initiation protein (ATCC9714PCS11_00101), the plasmid-partitioning proteins ParA (ATCC9714PCS11_ 00021) and ParB (ATCC9714PCS11_00011), a putative topoisomerase (ATCC9714PCS11_00271), a RecA recombinase (ATCC9714PCS11_00141), a resolvase (ATCC9714 PCS11_00561) and a helix-destabilising single-stranded DNA-binding protein (ATCC9714PCS11_00391). pCS1-1 also encodes a Type IV Secretion-System DNA conjugation protein (ATCC9714PCS11_00311) and a Type IV Secretion-System coupling DNA-binding domain protein (ATCC9714PCS11_00351), which may aid or enable conjugative transfer of the plasmid. Also found is a secreted collagen-binding protein (ATCC9714PCS11_00241), and a second copy of sortase (one is present on the chromosome) 
Table 1 C. sordellii strains used in this study

\begin{tabular}{|c|c|c|c|}
\hline Strain & Source & Associated infection or pathology & ENA Accession numbers \\
\hline R32977 & ARU (UK) & Knee Replacement & [CELC01000001-CELC01000028[ \\
\hline R32921 & $\mathrm{ARU}(\mathrm{UK})$ & Death During Pregnancy & [CEKV01000001-CEKV01000024[ \\
\hline R32668 & ARU (UK) & Wound Infection & [CEKY01000001-CEKY01000028[ \\
\hline R32462 & ARU (UK) & Endo-Cervical Discharge & [CEMX01000001-CEMX01000022[ \\
\hline R31809 & ARU (UK) & Traumatic Knee Injury & [CEKW01000001-CEKW01000031[ \\
\hline R30684 & ARU (UK) & Calf Abscess & [CELE01000001-CELE01000028] \\
\hline R29426 & ARU (UK) & Sudden Death & [CEMY01000001-CEMY01000040] \\
\hline R28058 & ARU (UK) & Crushed Hand & [CEKZO1000001-CEKZ01000028] \\
\hline R27882 & ARU (UK) & Knee Amputation & [CELB01000001-CELB01000031] \\
\hline R26833 & ARU (UK) & Blood Culture from Diabetic & [CELG01000001-CELG01000030] \\
\hline W2967 & VLA (UK) & Veterinary Isolate & [CELJ01000001-CELJ01000026] \\
\hline W10 & VLA (UK) & Veterinary Isolate & [CELH01000001-CELH01000051] \\
\hline W2922 & VLA (UK) & Veterinary Isolate & [CELK01000001-CELK01000153] \\
\hline W2945 & VLA (UK) & Veterinary Isolate & [CELI01000001-CELI01000027] \\
\hline W2946 & VLA (UK) & Veterinary Isolate & [CENA01000001-CENA01000080] \\
\hline W2948 & VLA (UK) & Veterinary Isolate & [CELF01000001-CELF01000026] \\
\hline W2975 & VLA (UK) & Veterinary Isolate & [CEKX01000001-CEKX01000030] \\
\hline W3025 & VLA (UK) & Veterinary Isolate & [CELA01000001-CELA01000022] \\
\hline W3026 & VLA (UK) & Veterinary Isolate & [CELD01000001-CELD01000043] \\
\hline JGS444 & ISU (USA) & Myonecrosis, Bovine & [CDNJ01000001-CDNJ01000028] \\
\hline JGS445 & ISU (USA) & Myonecrosis, Bovine & [CDNU01000001-CDNU01000031] \\
\hline JGS6382 & ISU (USA) [14] & Myonecrosis, Bovine & [LN681234-LN681235] \\
\hline JGS6956 & ISU (USA) & Veterinary Isolate & [CDNN01000001-CDNN01000034] \\
\hline JGS6961 & ISU (USA) & Veterinary Isolate & [CDNI01000001-CDNI01000027] \\
\hline ATCC9714 & ATCC (USA) [1] & Oedema & [LN679998- LN680000] \\
\hline DA108 & UMich (USA) [15] & Post-Partum Endometritis. & [CDNR01000001-CDNR01000036] \\
\hline SSCC33589 & UWA (AUS) & Blood Isolate & [CDNV01000001-CDNV01000026] \\
\hline SSCC42239 & UWA (AUS) & Blood Isolate & [CDNH01000001-CDNH01000029] \\
\hline SSCC26591 & UWA (AUS) & Blood Isolate & [CDNX01000001-CDNX01000035] \\
\hline SSCC37615 & UWA (AUS) & Blood Isolate & [CDNO01000001-CDNO01000031] \\
\hline SSCC18838 & UWA (AUS) & Blood Isolate & [CDNK01000001-CDNK01000030] \\
\hline SSCC18392 & UWA (AUS) & Blood Isolate & [CDNE01000001-CDNE01000027] \\
\hline SSCC35109 & UWA (AUS) & Blood Isolate & [CDNF01000001-CDNF01000036] \\
\hline SSCC33587 & UWA (AUS) & Blood Isolate & [CDNW01000001-CDNW01000028] \\
\hline SSCC32135 & UWA (AUS) & Blood Isolate & [CDNQ01000001-CDNQ01000031] \\
\hline UMC1 & OU (USA) [16] & Allograft Isolate & [CDNM01000001-CDNM01000035] \\
\hline UMC2 & OU (USA) [16] & Allograft Isolate & [CDLK01000001-CDLK01000054; LN681233] \\
\hline UMC164 & OU (USA) [16] & Allograft Isolate & [CDPO01000001-CDPO01000024] \\
\hline UMC178 & OU (USA) [16] & Allograft Isolate & [CDNP01000001-CDNP01000032] \\
\hline UMC4401 & OU (USA) [16] & Allograft Isolate & [CDNS01000001-CDNS01000027] \\
\hline UMC4404 & OU (USA) [16] & Allograft Isolate & [CDNY01000001-CDNY01000030] \\
\hline
\end{tabular}


Table 1 C. sordellii strains used in this study (Continued)

\begin{tabular}{|c|c|c|c|}
\hline E204 & MU (AUS) & Clinical Isolate & [CDNL01000001-CDNL01000025] \\
\hline R15892 & ARU (UK) & Clinical Isolate & [CEKU01000001-CEKU01000032] \\
\hline JGS6364 & ISU (USA) & Myonecrosis, Bovine & [CDL01000001-CDLJ01000026; LN681232] \\
\hline
\end{tabular}

which may anchor certain plasmid-encoded secreted proteins to the cell wall.

An equivalent plasmid termed pCS1-3 was found in JGS6382, the only strain in our collection that carries $t c s H$. After assembly of pCS1-3 by PCR, we found that the genes $t c s L, t c s H$ and $t c s E$ are arranged identically to those in the $t c s L^{+} / t c s H^{+}$strain VPI9048 (Fig. 2) [17]. Thus the $t c s L$ and $t c s E$ genes are in the same orientation, but $t c s H$ and $t c s R$ are encoded on the opposite strand. This orientation of the PaLoc genes is different to that found in C. difficile where the homologous PaLoc genes $t c d R, t c d B, t c d E$ and $t c d A$ are in the same orientation. No homologue of $t c d C$, a negative regulator of toxin expression in C. difficile [18] was found in any of the $C$. sordellii isolates in our study. Though pCS1-3 is a similar size to pCS1-1 (106 kb) and contains a similar number of ORFs (94 compared to 90 on pCS1-1) it contains several differences in addition to the presence of fulllength $t c s H$ within the PaLoc. 29 ORFs are absent from pCS1-3 relative to pCS1-1, including 2 universal stress proteins and all genes encoding anaerobic sulphite reductase subunits. However, 32 ORFs are present on

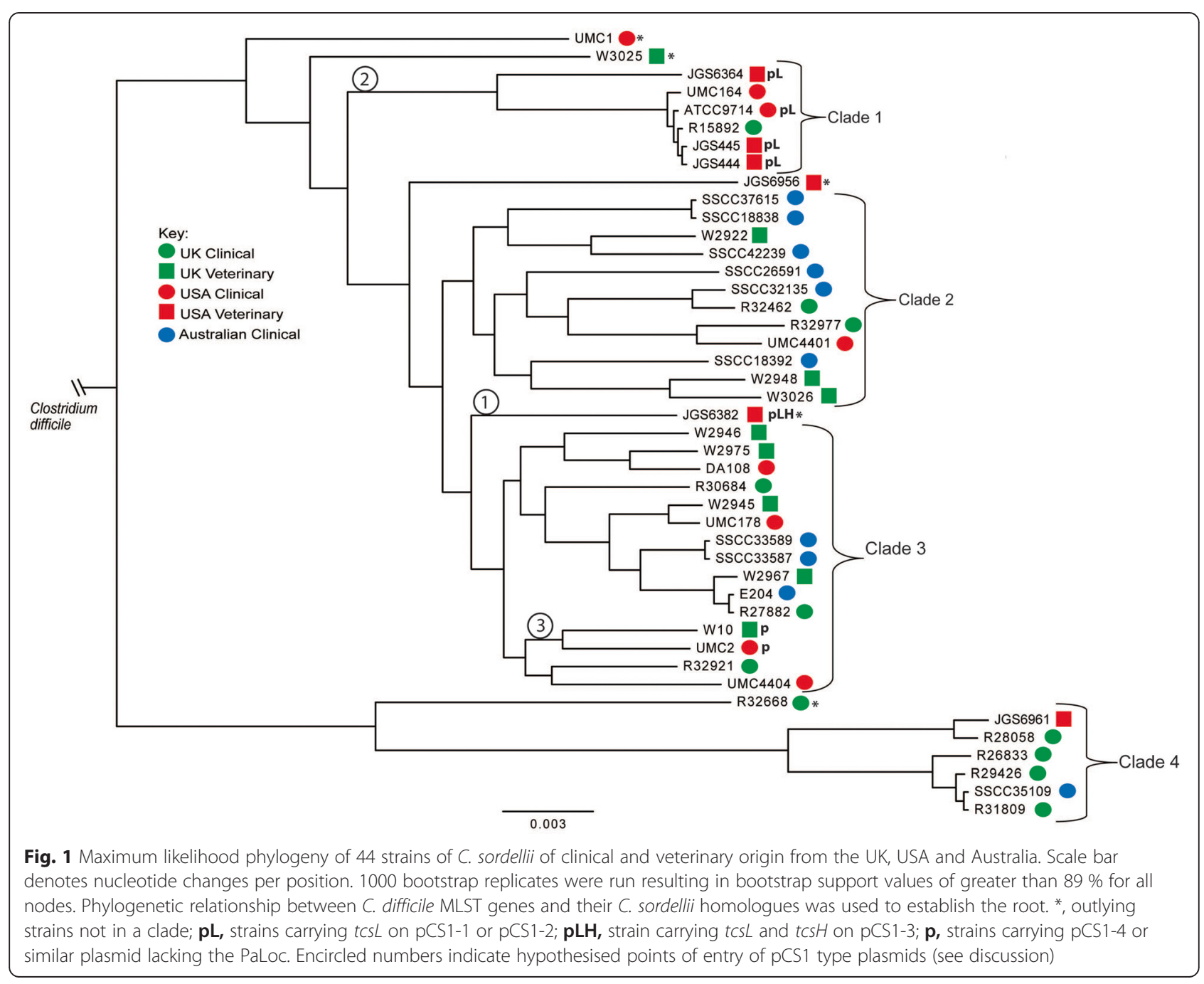




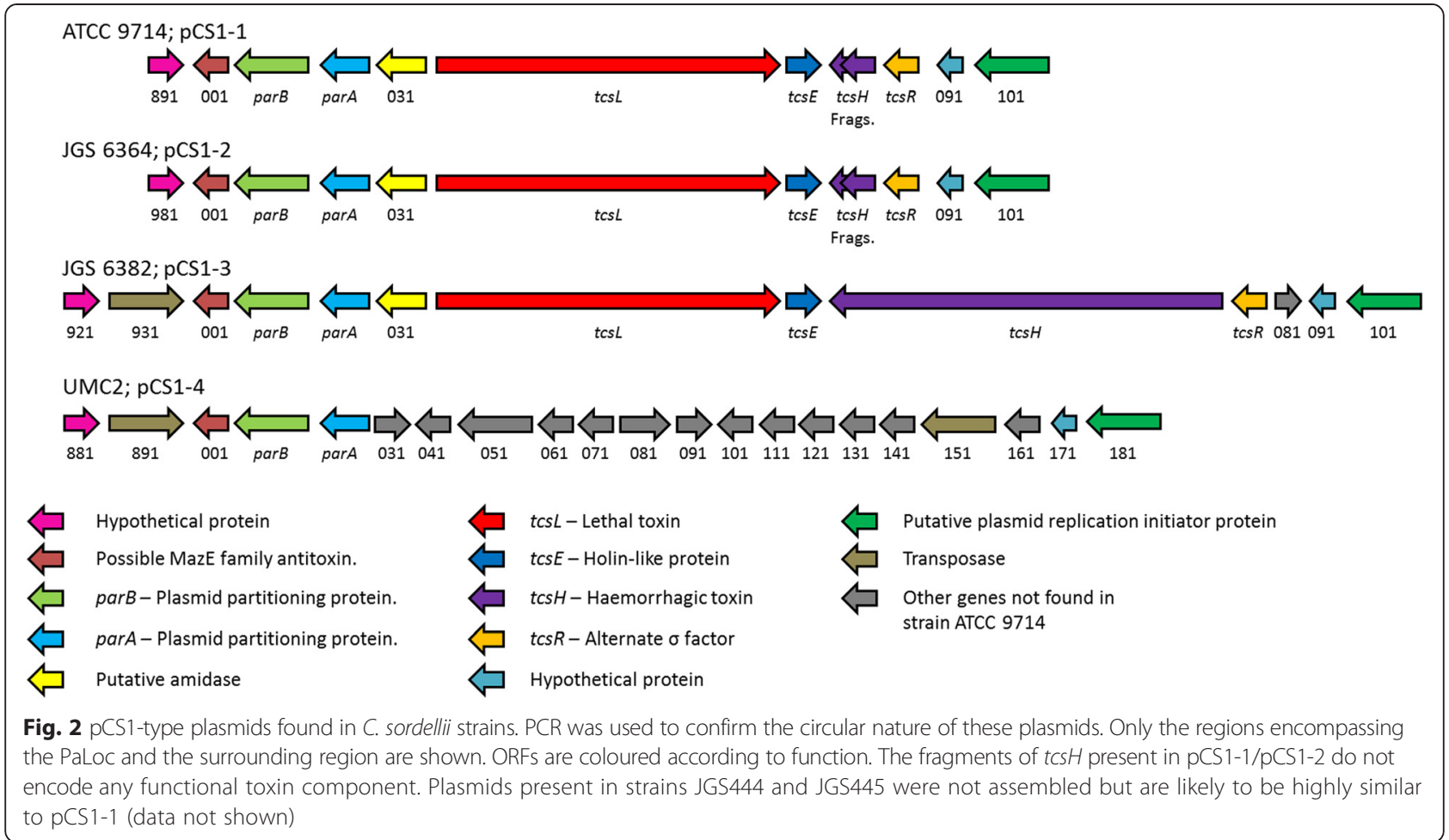

pCS1-3 that are not found on pCS1-1, including an additional copy of a resolvase and three transposase genes. Analysis of the sequence of C. sordellii VPI9048 [17] reveals that most ORFs from pCS1-3 are also present, suggesting that VPI9048, and potentially other $t c s H^{+}$ strains, contain a highly related plasmid.

Visual examination of the genomes of the $t c s L^{+} / t c s H^{-}$ strains JGS444 and JGS445 suggests that they each carry plasmids highly similar to pCS1-1, though these plasmids were not fully assembled by PCR. Examination of the genome of the $t c s L^{+} / t c s H^{-}$strain JGS6364 showed the presence of a plasmid similar to pCS1-1; however, pulsed field gel electrophoresis showed the plasmid in JGS6364 to be noticeably larger than pCS1-1 (Fig. 3), which prompted us to assemble the plasmid by PCR to examine the differences between it and pCS1-1. We designated the plasmid present in JGS6364 as pCS1-2 which, at $117.3 \mathrm{~kb}$, is $13.5 \mathrm{~kb}$ larger than pCS1-1. The arrangement of genes within the PaLoc of pCS1-2 was identical to in pCS1-1 (Fig. 2), but several major differences were seen elsewhere on the plasmid. Several genes are lost relative to pCS1-1, including ones encoding a transcriptional regulator and a cold shock protein. Several genes are present which are not found on pCS1-1, including 23 found on a $21 \mathrm{~kb}$ insertion. These include various putative lantibiotic biosynthesis genes and a putative lantibiotic-binding component of an $\mathrm{ABC}$ transporter. It would be of interest to determine if JGS6364 does indeed produce a lantibiotic, because as far as we are aware lantibiotic synthesis has not previously been identified in the Clostridia.
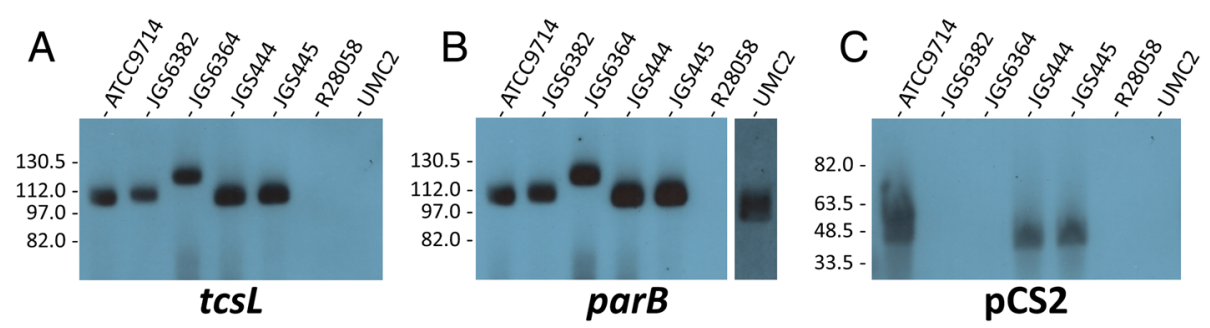

Fig. 3 Southern hybridisation analysis of pulsed-field gels to confirm the presence of pCS1- and pCS2-type plasmids in C. sordellii strains. (A) A blot of a pulsed-field gel using C. sordellii genomic DNA-containing agarose plugs digested with B/pl (to linearise pCS1-type plasmids), hybridised with a tCSL specific probe; (B) the blot from panel A was stripped and reprobed with a probe specific for parB from pCS1-1; (C) a blot of a separate pulsed-field gel using purified uncut C. sordellii genomic DNA, hybridised with a probe specific for ATCC9714PCS2_00141 from pCS2. The migration of molecular size markers is indicated on the left, and the C. sordellii isolate corresponding to each lane is indicated above 
Two strains in our collection that lack tcsL and $t c s H$ (UMC2 and W10, indicated with a $\mathbf{p}$ in Fig. 1) were also found to carry plasmids similar to pCS1-1. The $100 \mathrm{~kb}$ plasmid in strain UMC2 was assembled by PCR and designated pCS1-4. pCS1-4 is highly similar to the pCS1 plasmids (Fig. 2), however the PaLoc genes are absent and in their place a series of genes is present including a transposase. Interestingly, in $\mathrm{pCS} 1-3$ and $\mathrm{pCS} 1-4$, upstream of a conserved putative MazE family antitoxin gene a second transposase gene is found.

During manual assembly of the ATCC9714 genome a second circular element, pCS2, was also identified. pCS2 $(37.1 \mathrm{~kb})$ is smaller than $\mathrm{pCS} 1-1$ and its relatives, and contains 52 ORFs. pCS2 is clearly phage-derived, with 20 of its 52 ORFs annotated as phage genes, including multiple genes encoding structural phage components. It also contains two genes potentially involved in replication (soj/parA (ATCC9714PCS2_00031) and a replication initiator protein (ATCC9714PCS2_00011)). Whether this element represents a phage or plasmid is unknown.

\section{Confirmation of plasmid presence by PFGE and Southern blot analysis}

Southern hybridisation analysis of pulsed-field gels was performed to confirm the presence of pCS1-type plasmids among C. sordellii isolates (Fig. 3). Examination of the genome sequences of the five toxigenic $C$. sordellii isolates and UMC2 indicated the presence of only a single $B l p \mathrm{I}$ restriction site on all pCS1-type plasmids. To linearise and therefore better visualise the pCS1-type plasmids, C. sordellii genomic DNA-containing plugs were digested with $B l p I$ before being subjected to pulsed-field gel analysis. All hybridisation bands observed for BlpI digested samples in both the tcsL and parB blots (Fig. 3) were also seen in the uncut samples (data not shown), confirming that the bands shown in Fig. 3 represent linearised versions of the pCS1-type plasmids. In the tcsL blot, a band corresponding to the size of the pCS1-1 plasmid (103 kb) can be seen (Fig. 3A). Bands of equal or higher molecular weight are evident from other toxigenic $C$. sordellii isolates, however, no bands were observed for the non-toxigenic isolates R28058 and UMC2. The size of the pCS1-type plasmids in JGS444 and JGS445 appears to be equal to that of the plasmid in ATCC9714, supporting genome sequence data that indicates these strains carry plasmids highly similar to pCS1-1. The band corresponding to pCS1-3 in JGS6382 appears to be of slightly higher molecular weight than that of pCS1-1, which agrees with the size of pCS1-3 (106 kb) based on genome sequence analysis. The band corresponding to pCS1-2 from JGS6364 is of higher molecular weight than all other pCS1-type plasmids, as discussed earlier. An identical hybridisation pattern can be seen for the parB blot in comparison to the tcsL blot for the toxigenic $C$. sordellii isolates and the negative control R28058, however, a band can also be seen in the parB blot for UMC2 (Fig. 3B), confirming the presence of plasmid pCS1-4 in this strain. A probe for a locus on the pCS2 plasmid (ATCC9714PCS2_00141) was used to confirm the presence of this plasmid in ATCC9714 and to determine if it is present in the other C. sordellii isolates tested. A band can be seen in uncut ATCC9714 genomic DNA in the pCS2 blot, indicating that this plasmid is present in this strain (Fig. 3C). Similar bands can be observed from both JGS444 and JGS445, indicating that pCS2 or a related plasmid is also present in these strains, which may be expected as these isolates are phylogenetically related to ATCC9714 (Fig. 1). Indeed, visual analysis of all 44 genome sequences suggests pCS2 is also found in the closely related clade 1 strains UMC164 and R15892. The pCS2 probe did not hybridise to the genomic DNA from any of the remaining $C$. sordellii isolates in the panel of strains tested indicating that this plasmid is not present among these strains.

\section{The ATCC9714 genome}

The sequence of strain ATCC9714 was refined, producing a high quality reference genome. 3282 ORFs are annotated on the chromosome, together with 90 on pCS1- 1 and 52 on pCS2 giving a total of 3424 . This is in line with other Clostridial species - significantly more than $C$. perfringens or C. tetani (strains 13 and E88 respectively have approximately 2660 and 2580 chromosomal ORFs) but fewer than C. difficile strain 630, which has approximately 3680. ATCC9714 contains the fewest ORFs of the strains in our collection, the most being 3957 ORFs in strain UMC2, and the average being 3459 ORFs. However, these figures are preliminary, as the genomes other than ATCC9714 have undergone little improvement to reduce contig number, which would almost certainly reduce the number of ORFs identified. Further analysis would also allow the identification and assembly of plasmids.

\section{Putative virulence factors}

168 putative secreted proteins were identified in the ATCC9714 genome (Additional file 1), six of which are encoded by pCS1-1 and one by pCS2. Of the 168 ORFs, 66 are predicted to encode lipoproteins, including one encoded on pCS1 and a second on pCS2. There are also nine predicted cell wall proteins, which contain 3 adjacent S-layer homology (SLH) domains, which can non-covalently attach proteins to the cell wall [19]. This attachment requires pyruvylation of the cell wall by the protein CsaB [19], which is encoded on the chromosome (ATCC9714_28031). The $c s a B$ gene is located just downstream of two SLH domaincontaining genes, ATCC9714_28071 (function unknown) and ATCC9714_28081 (a putative amidase). Based on their 
location adjacent to $c s a B$, by analogy to other species, e.g. Bacillus anthracis [19], these SLH-domain proteins might be constituents of an S-layer.

Four probable exotoxins were identified: a secreted collagenase colA (ATCC9714_10061), the cholesterol-dependent cytolysin (CDC) $s d l$ (sordellilysin) (ATCC9714_18311), the neuraminidase nanS (ATCC9714_16161) and phospholipase C (csp, ATCC9714_31321). The $s d l$ gene is highly conserved between all strains sequenced ( $\geq 97 \%$ protein sequence identity). The encoded protein shows strong similarity to perfringolysin $\mathrm{O}$ from $C$. perfringens, and all residues identified as being of functional or structural importance in perfringolysin $\mathrm{O}[20,21]$ are conserved in sordellilysin, including the undecapeptide. It has previously been shown that sordellilysin functions as a CDC, but that not all strains containing the $s d l$ gene produce SDL under standard conditions. Such strains include ATCC9714, UMC164 and UMC178, all part of this collection [16].

$\mathrm{NanS}$ is a homologue of the small C. perfringens neuraminidase NanH [22]. Multiple catalytically and structurally important residues and motifs have been identified, including four structurally important 'Asp-Boxes', all of which are conserved in the ATCC9714 NanS. One important difference between C. sordellii NanS and C. perfringens $\mathrm{NanH}$ is that $\mathrm{NanH}$ is located in the cytoplasm, while NanS possesses a signal sequence and has been shown to be secreted, and indeed to cause/contribute to the leukemoid reaction characteristic of severe $C$. sordellii infection [23]. NanS is extremely well conserved between all 44 strains in our collection ( $\geq 97 \%$ protein sequence identity); however, two strains, the closely related SSCC37615 and SSCC18838 from clade 2, possess a $\mathrm{G}=>$ A point mutation resulting in a Asp162Asn mutation. Asp162 is the equivalent of NanH Asp144, which has been shown to be necessary for correct folding of the protein [22]. NanS produced by these strains may therefore not be fully functional.

Csp is closely related to several Clostridial phospholipases $\mathrm{C}$, including the Clostridium bifermentans phospholipase $C$ and C. perfringens $\alpha$-toxin. C. sordellii ATCC9714 Csp has been shown to be enzymatically active, but less active than $\alpha$-toxin, less haemolytic and, alone, to be nontoxic to mice [24]. All known residues essential for enzymatic activity and structural maintenance are conserved within the C. sordellii protein [24]. Strains have previously been identified which do not produce Csp, although the majority do [16]. Four closely related strains from clade 4 (R26833, R29426, SSCC35109 and R31809) all possess a $\mathrm{T}=>$ A point mutation in codon 50 , resulting in the formation of a premature stop codon, which will preventing production of Csp. JGS6382 has an N-terminal truncation resulting in the absence of a signal sequence, meaning it may produce Csp but will not secrete it. All other strains are predicted to produce functional Csp, though JGS6956 $\mathrm{Csp}$ has a slight $\mathrm{C}$-terminal truncation. The $\mathrm{C}$-terminus of $\alpha$-toxin is believed to bind $\mathrm{Ca}^{2+}$ ions [25]; the truncation found in JGS6956 Csp would likely render it unable to bind $\mathrm{Ca}^{2+}$, which may affect its function.

We are unaware of any prior studies on ColA, the $C$. sordellii collagenase. Comparison with ColG from C. histolyticum, the only Clostridial collagenase for which a structure is available, shows some similarity (33\% sequence identity) and the conservation of several important residues, including all those which bind the catalytic $\mathrm{Zn}^{2+}$ ion and others important in substrate recognition. However, mature ColA is 79 amino acids shorter than ColG, and the PKD-like domain, important in ColG substrate binding [26], is entirely absent from ColA, raising questions as to how it binds its substrate. However, ColA is well conserved between all strains of $C$. sordellii in our collection ( $\geq 95 \%$ sequence identity).

Two putative virulence factors were identified which have predicted functions in immune evasion. ATCC9714 13801 encodes aureolysin, a secreted metalloprotease first identified in Staphylococcus aureus which cleaves complement protein $\mathrm{C} 3$, preventing complement activation during infection [27], and the anti-microbial peptide LL-37 [28], aiding evasion of the host immune system during infection. ATCC9714_09801 contains a Mac-1 domain, which is found in the Streptococcus pyogenes protein IdeS and contains a protease function which specifically cleaves human IgG, aiding immune evasion [29].

Two putative adhesins were also identified on the chromosome. The first is a secreted collagen-binding protein of $>140 \mathrm{kDa}$ (ATCC9714_22821/22831), containing at least $7 \mathrm{CnaB}$ repeats. Due to the length and repetitive nature of this gene we were unable to fully sequence it, so it is split across two contigs. The second adhesin is encoded by another large gene (ATCC9714_14191) which contains two discoidin domains; in eukaryotes discoidin domain receptors (DDRs) bind collagen [30]. ATCC9714 also contains two Type IV Pili (T4P) gene clusters (ATCC9714_01091-ATCC9714_01201 and ATCC9714_ 02121-ATCC9714_02151). T4P are able to promote adhesion both between bacterial cells in biofilms and between bacterial and mammalian cells, and thus commonly act at as virulence factors [31]. These putative adhesins and T4P clusters are conserved in every strain sequenced, as are genes encoding a full flagella apparatus.

It has previously been shown that many, but not all, strains of $C$. sordellii possess urease activity [32, 33]. Urease is a nickel-containing metalloenzyme that hydrolyses urea into ammonia and carbonic acid. It acts as a virulence factor in several bacterial and fungal pathogens, including Helicobacter pylori and Klebsiella species [34], though there is no evidence that urease acts as a virulence factor in C. sordellii. We found complete urease operons in all strains in our collection except SSCC26591. 
The operon comprises 8 genes, ureABCIEFGD, and is located on the chromosome immediately upstream of nanS (ATCC9714_16171 - 16241). To our knowledge no other Clostridia carry chromosomal urease genes, though a small minority of strains of $C$. perfringens are known to carry urease genes borne on a plasmid [35]. The urease enzyme itself comprises UreABC proteins that initially form an apoenzyme that is activated by the UreDFG complex. UreE is a Ni-binding chaperone and UreI is a urea transporter. All genes are highly conserved between strains ( $\geq 87 \%$ protein sequence conservation from every gene). UreC contains the enzyme's active site and all previously identified Ni-binding/catalytically active residues are conserved across all UreCs encoded in our collection [36].

\section{Sporulation and germination}

All clostridial species are able to form metabolically inactive, stress-resistant spores [37]. Sporulation is controlled by the master regulator spoOA, a transcriptional regulator which controls expression of sporulation genes [38]. As expected, spoOA is present in C. sordellii ATCC9714 (ATCC9714_26501). In Bacillus subtilis the proteins SpoOF and Spo0B form a phosphorelay from the kinase KinA, resulting in downstream Spo0A phosphorylation and activation [38]. C. difficile lacks the spoOF and $\mathrm{spoOB}$ genes, and $\mathrm{SpoOA}$ is instead directly phosphorylated by two histidine kinases: CD630_1579 and CD630_2492 [39]. Two homologues of CD630_1579 have been identified in ATCC9714: ATCC9714_07111 and ATCC9714_16961, suggesting a similar mode of regulation and activation of Spo0A in C. sordellii as in C. difficile.

The majority of the sporulation machinery appears to be conserved between $C$. difficile and $C$. sordellii, and it is likely that the sporulation process in C. sordellii is similar to that in C. difficile. Also highly similar to $C$. difficile are the spore-coat proteins found in C. sordellii. 8 proteins have been confirmed as constituents of the spore-coat in C. difficile strain 630: CotABDEFG, CotCB and SodA [40]. Likely homologues have been identified in C. sordellii ATCC9714 of CotA (ATCC9714_1722), CotB (ATCC9714_05091), CotD (ATCC9714_05851), CotE (ATCC9714_12131), and SodA (ATCC9714_20791). No specific homologues have been identified for CotCB, CotF or CotG; however, CotCB and CotG are highly similar to CotD and are both also closely homologous to ATCC9714_05851. CotF is homologous to ATCC9714_ 05861, though this gene appears more closely related to the C. difficile gene CD630_2400, currently annotated as the putative spore-coat gene, $\cot J B 2$, which is very similar to $\cot F$ but is not yet uncharacterised. It thus seems likely that the C. sordellii spore-coat is similar to that of $C$. difficile, but potentially simpler; where $C$. difficile possesses 2 or 3 paralogues of certain genes $(\cot C B, \cot D$ and $\cot G$, or $\cot F$ and $\cot J B 2), C$. sordellii possesses only one equivalent gene. One potentially significant difference between $C$. difficile and C. sordellii spores has been identified. C. difficile 630 has three bclA genes, homologues of which in B. anthracis encode glycoproteins forming filaments on the surface of the spore [41]. No such homologues were identified in C. sordellii ATCC9714.

Spores are able to germinate and produce vegetative cells. Germination of both $C$. difficile and $C$. sordellii spores is known to be initiated by spore surface receptors that recognise and bind mammalian bile salts [42]. The only known bile salt receptor in $C$. difficile is the serine protease CspC [43], a homologue of which is also found in C. sordellii ATCC9714 (ATCC9714_11661).

\section{Discussion}

Our genomic analysis of a collection of C. sordellii strains reveals several important findings related to the phylogeny of the species and to the pathogenesis of disease. Phylogenetic analysis reveals the presence of four clades within our strain collection, which includes strains from diverse geographical locations and from infections of both veterinary and medical origin. None of the four assigned clades is specifically associated with either clinical or veterinary disease; rather, both clinical and veterinary isolates are found in each, suggesting that no group of strains has adapted to any specific host. Also, no clade is specifically associated with any country of origin. Given the relative geographical isolation of the USA, UK and Australia, one might have expected strains located in each nation to have diverged. The fact that strains sourced from each country are closely related to strains sourced from the others may suggest recent transfer of strains between countries. How this has occurred remains unclear, though one likely possibility is through human activity such as travel or export of livestock.

We found that only a small minority of $C$. sordellii strains contain the LCC genes $t c s L$ and $t c s H$. A similar but smaller study in 2006 resulted in similar findings, with only 1 of 14 isolates analysed containing tcsL and none containing tcs $H$ [16]. In our study, 5 out of 44 strains encode $t c s L$, though two of those (JGS444 \& JGS445) are highly related, suggesting they may in fact be the same strain collected from different sources, possibly during a minor outbreak, and only one carries $t c s H$. The 44 strains sequenced here provide a likely representation of the species as a whole, suggesting that only a minority of strains isolated carry $t c s L$ and that $t c s L^{+} / t c s H^{+}$ strains are rare. In support of this, a recent study of 52 isolates from a single US strain collection found only 3 to contain $t c s H$ (by PCR), although the number containing $t c s L$ was not reported [44]. 
It is highly relevant that the LCC genes are plasmid encoded. Strain UMC164 was previously shown to carry $t c s L$, and indeed to produce the toxin [16], but the isolate we analysed lacks $t c s L$ and the other PaLoc genes. This suggests that the original isolate of UMC164 carried a pCS1-type plasmid but this was lost during subculturing. Indeed, in a recent paper describing clinical cases of C. sordellii infection, Bouvet et al. [45] speculated that the LCC genes are encoded on a mobile genetic element which is lost upon sub-culture. They report isolating strains of $C$. sordellii lacking the $t c s L$ gene from clinical cases where toxin activity had been demonstrated in intestinal contents. It is therefore likely that the majority of $C$. sordellii infections are due to strains containing a PaLoc on pCS1-type plasmids, but that these genetic elements are often lost from strains during subculture. Thus the PaLoc and its associated plasmid may be present in a considerably larger proportion of naturally occurring $C$. sordellii strains than indicated by previous studies and indeed our own genome survey. The availability of the DNA sequences for the plasmid and toxin genes will allow early analysis of clinical samples by PCR which could identify toxin genes before they are lost upon subculture.

A previous study employed genetic inactivation of tcsL in ATCC9714 to probe the requirement of TcsL to establish disease in two mouse models of infection [13]. In both models the wild type strain caused severe disease while the tcsL mutant was avirulent, demonstrating that TcsL is an essential virulence factor in these models, and other studies have also indicated that TcsL is required for the establishment of toxic shock associated with severe $C$. sordellii infections, though non-toxigenic strains are capable of causing less severe infections [14, 15]. Given that the majority of strains in our collection are from clinical/veterinary cases of disease, it seems likely that a significant number of them might have originally harboured a pCS1-type plasmid, which has been lost upon laboratory subculture. This would include UMC164 (as stated above) and any which were associated with severe infections/toxic shock syndrome. From our phylogenetic tree, strain R15892 has probably lost a pCS1-type plasmid, being closely related to the $t c s L^{+}$strains ATCC9714 and JGS444/445. It is a matter of speculation why some strains lose their toxin-encoding plasmid while others don't. Possibilities include the pCS-1 plasmids being stable variants of the highly unstable plasmids or the unstable plasmids having a distinct replication or partition apparatus conferring instability under laboratory conditions.

We should not assume that all strains lacking the PaLoc were once toxigenic and have since lost their pCS1-type plasmids. The 'UMC' strains were obtained from cadavers and had not caused infection in their host humans [16], meaning some of them at least might be avirulent. It is also possible that some of the strains described here may have caused infections despite lacking tcsL. It has been estimated that only two-thirds of reported cases of $C$. sordellii infection are associated with toxic shock syndrome, and this could even be an overestimate with less dramatic cases being overlooked [14]. At least one of the non-toxigenic strains, DA-108, was derived from a clinical infection not associated with toxic shock syndrome [15], while strain W10 was isolated from a veterinary infection but appears to carry a pCS1-4-like plasmid; i.e. it still carries a pCS1-type plasmid but lacks tcsL. All strains in our study contain other putative virulence factors including the cholesteroldependent cytolysin sordellilysin $(s d l)$, neuraminidase (nanS), phospholipase $\mathrm{C}(\mathrm{csp})$ and collagenase (colA). Cases of $C$. sordellii invasive disease not linked to toxic shock syndrome and caused by apparently LCC-negative strains have been described [14, 15, 46, 47]. It is therefore possible that a number of these strains were nontoxigenic at the time of infection and were associated with less severe disease (e.g. R30684 was isolated from an abscess).

In $C$. difficile, the toxin genes $t c d A$ and $t c d B$ are chromosomally localised and strains carrying both toxin genes $t c d A$ and $t c d B$ constitute the majority of strains isolated from $C$. difficile infections, with those carrying only $t c d B$ comprising around 5-10\% of clinical isolates $[48,49]$. Non-toxic $C$. difficile strains are routinely isolated from patients [49], but it remains to be demonstrated that such $t c d A^{-} / t c d B^{-}$strains are pathogenic. Interestingly, to date no naturally occurring $C$. difficile $t c d A^{+} / t c d B^{-}$strains or C. sordellii $t c s H^{+} / t c s L^{-}$strains have been found. The relative orientations of the C. sordellii toxin genes and their regulatory genes tcsE and $t c s R$ are distinct to those found in $C$. difficile, while no homologue of $t c d C$, which negatively regulates toxin production in C. difficile [18], is present.

Our findings raise the question of the evolution of the LCC genes. Our phylogenetic analysis suggests that a pCS1-like plasmid has entered strains of C. sordellii on at least 3 occasions (see Fig. 1). One possible chronology is as follows: pCS1-3, bearing both $t c s L$ and $t c s H$, first entered C. sordellii (indicated by " 1 " in Fig. 1). On a second occasion, "2", pCS1-3 entered into the ancestor strain of clade 1, which includes ATCC9714. Herein a rearrangement occurred resulting in the loss of the vast majority of $t c s H$ and the eventual formation of pCS1-1 and pCS1-2. On a third occasion, "3", pCS1-3 could have entered an ancestor of strains UMC2 and W10 and undergone a rearrangement resulting in the loss of the entire PaLoc, forming pCS1-4. Of course, several other potential chronologies exist, and given the possibility that multiple strains may have once carried such a plasmid and 
since lost it this analysis may be lacking. Whole genome sequencing of other known $t c s L^{+}$strains would allow improvement of this tree and greater understanding of the distribution of these plasmids throughout the species.

Another question is the origin of the LCC genes in C. sordellii, and whether the plasmid bearing them is passed directly between strains of $C$. sordellii or is transferred via an external intermediate. It has recently been shown that $C$. difficile LCC genes can undergo horizontal transfer between strains, a process likely mediated via conjugative transposons [50], but it is not yet known whether the LCC genes of $C$. sordellii can be transferred between strains via plasmid transfer.

\section{Conclusion}

Our sequencing of C. sordellii genomes reveals a surprising deficit of LCC genes, that where present they are plasmid-localised, and evidence of plasmid loss from certain strains. This suggests that the LCC genes may be more widespread within the species than the screening of isolates initially indicates. Less severe infections may be caused by LCC-negative strains where virulence is facilitated by other virulence factors, some of which we identified in strain ATCC9714. Although a clade structure was identifiable in our collection of strains, these did not divide by geographical region or by infected host species. The high-quality reference genome sequence of $C$. sordelllii ATCC9714 will enable further research on this important pathogen. Finally, comparison of C. sordelliii sequences with those of other pathogenic clostridia will help to characterise the wide range of strategies that the clostridia use to survive and proliferate in man and animals.

\section{Methods}

\section{Bacterial strains and growth conditions}

C. sordellii strains were grown in BHIS broth $(37 \mathrm{~g} / \mathrm{L}$ Brain-Heart Infusion, $5 \mathrm{~g} / \mathrm{L}$ yeast extract, $1 \mathrm{~g} / \mathrm{L}$ L-Cysteine) or on BHIS agar (as above, with $15 \mathrm{~g} / \mathrm{L}$ agar) in an anaerobic cabinet (Don Whitley Scientific) in an atmosphere of $80 \% \mathrm{~N}_{2}, 10 \% \mathrm{CO}_{2}$ and $10 \% \mathrm{H}$. A full list of strains used in this study can be found in Table 1 .

\section{DNA extraction and sequencing}

Genomic DNA was prepared by growth of $25 \mathrm{ml}$ overnight cultures of $C$. sordellii in BHIS broth. Cells were harvested by centrifugation $(4000 \mathrm{x} \mathrm{g}, 10 \mathrm{~min})$. Cells were frozen at $-80{ }^{\circ} \mathrm{C}$ for $1 \mathrm{~h}$ then re-suspended in $400 \mu \mathrm{l}$ lysis buffer ( $200 \mathrm{mM} \mathrm{NaCl}, 50 \mathrm{mM}$ EDTA, $20 \mathrm{mM}$ Tris- $\mathrm{HCl}$, $\mathrm{pH}$ 8). Lysozyme $(2 \mathrm{mg} / \mathrm{ml})$ and RNase A $(0.1 \mathrm{mg} / \mathrm{ml})$ were added and the suspension incubated at $37{ }^{\circ} \mathrm{C}$ for $2 \mathrm{~h}$. Proteinase K $(0.5 \mathrm{mg} / \mathrm{ml})$ and SDS $(1 \%)$ were added and the suspension incubated at $50{ }^{\circ} \mathrm{C}$ for $1 \mathrm{~h}$. DNA was then extracted using phenol/chloroform, and precipitated by adding 2.5 volumes of ice-cold ethanol followed by overnight incubation at $-20{ }^{\circ} \mathrm{C}$. DNA was harvested by centrifugation at $17900 \mathrm{xg}$ for $5 \mathrm{~min}$ at $4{ }^{\circ} \mathrm{C}$, washed with $1 \mathrm{ml} 70 \%$ ethanol, centrifuged at $17900 \times \mathrm{g}$ for $5 \mathrm{~min}$ at $4{ }^{\circ} \mathrm{C}$ again and the supernatant removed. The pellet was air-dried at room temperature then resuspended in $\mathrm{H}_{2} \mathrm{O}$. Genomic DNA was sequenced on an Illumina Hi-Seq in a multiplex run of 48 samples.

\section{Assembly of genomes and annotation}

De novo assembly of the Illumina sequence data into contigs was carried out using VelvetOptimiser (Victorian Bioinformatics Consortium). The genome of ATCC9714 was then optically mapped [51] (see below), establishing the order and relative orientations of all contigs $>50 \mathrm{~kb}$. Gapfiller [52] (BaseClear) was then used to attempt to computationally close sequence gaps and iCORN (http:// icorn.sourceforge.net) to correct for any sequence errors. CDSs (Coding DNA Sequences) were identified using Prodigal [53] and functional annotation was transferred using four other clostridial genomes as references in an iterative process: C. difficile 630 (accession number AM180355); Clostridium botulinum Hall A (Proteolytic) (accession number AM412317); C. botulinum E3 Strain Alaska E43 (non-proteolytic) (accession number CP001078); Clostridium novyi NT (accession number CP000382) (in that order). The genome sequence was further improved using a combination of manual inspection of sequence data, PCR and comparison to the other $C$. sordellii strains and other clostridia. (For primer sequences, see Additional file 2). Subsequent mapping of the sequence data to the optical map allowed for validation of the genome assembly. Annotation to identify coding sequences, assign predicted functions and to predict RNA structures of the other C. sordellii strains was generated using Prokka [54].

\section{Optical mapping}

BglII was identified by OpGen enzyme selection software as an appropriate restriction enzyme with which to digest C. sordellii gDNA. $2 \mu \mathrm{l}$ gDNA was applied to an OpGen MapCard and run on an Argus system following manufacturer's protocols. The MapCard chambers were loaded with $\mathrm{JOJO}^{\mathrm{mm}}$ stain and OpGen enzyme, buffer and antifade, and the card cycled on the Argus ${ }^{\circ} \mathrm{MCP}$ (MapCard Processing Unit) for approximately $25 \mathrm{~min}$ at $37^{\circ} \mathrm{C}$. Following data collection, contig assembly was performed using Argus ${ }^{\circ}$ MapManager ${ }^{\mathrm{rm}}$; prior to assembly the mapset was filtered for minimum molecule size $>250 \mathrm{~kb}$, minimum fragments per molecule $>12$ and minimum molecule quality $>0.4$. The genome was initially assembled into a contiguous, circularized chromosome. Any regions of low coverage were improved using the "Find Hits" feature until depth of coverage reached 30x. The QC module was then used to perform a $\mathrm{QC}$ review to ensure all regions of map assembly were robust. The de novo sequence 
assembly was then aligned to the Optical Map using Argus Mapsolver. $89.8 \%$ of the de novo sequence assembly was aligned to the Optical Map with the remainder either contigs smaller than $50 \mathrm{~kb}$ which were too small to accurately align or plasmid sequence which was not incorporated into the Optical Map assembly.

\section{Generation of phylogenetic tree}

A core genome of C. sordellii was created using CD-HIT [55] to perform an initial clustering of the common genes shared between all 44 strains based on a $90 \%$ identity threshold followed by an all-against-all blast, the results of which were then input to a Markov Cluster Algorithm to perform the final clustering. In total 2712 genes were clustered to create the core C. sordellii genome. The sequences were concatenated and a nucleotide alignment was created using Muscle [56]. A maximum likelihood phylogeny was generated from the aligned sequence using FastTree version 2.1.3 [57] with the following settings: a Generalised Time-Reversible (GTR) model of nucleotide substitution and CAT approximation of the variation in rates across sites with 20 rate categories. BLASTN queries of the assembled C. sordellii genomes along with reciprocal annotation using different members of the order Clostridiales against the C. sordellii ATCC9714 genome revealed $C$. difficile to be the closest relative (86 \% BLASTN identity). C. difficile proved to be too diverse as an out-group to determine a meaningful phylogeny with $C$. sordellii using the methods described above. The root of the tree was therefore established by aligning the 7 MLST genes [58] of the C. difficile strain R20291 (Ribotype 027, Accession no. FN545816) with their corresponding $C$. sordellii orthologues (all present in every strain at $90 \%$ identity) and generating a phylogeny from the alignment using FastTree as described above. R20291 was chosen as it was the closest match to $C$. sordellii with a high-quality sequence.

\section{PCR}

PCR reactions were performed with KOD Hot Start DNA polymerase (Merck Millipore). Reactions were set up according to the manufacturer's instructions. $200 \mathrm{ng}$ of gDNA was used in each reaction which entailed an initial denaturation step $\left(94{ }^{\circ} \mathrm{C}, 2 \mathrm{mins}\right)$ followed by 30 cycles of: $94{ }^{\circ} \mathrm{C}, 15 \mathrm{~s}$; primer Tm minus $5^{\circ} \mathrm{C}, 30 \mathrm{~s} ; 68^{\circ} \mathrm{C}$, $1 \mathrm{~min} / \mathrm{kb}$ predicted product size. The primer Tms were calculated using https://ecom.mwgdna.com/services/webgist/ mops.tcl. PCR products were purified using a Qiagen QIAquick $^{\ominus}$ PCR Purification Kit. Screening for tcsL was performed using primers NF2362 and NF2363 and for tcsH using NF2351 and NF2352.

\section{Pulsed-field gel electrophoresis (PFGE)}

Growth of $C$. sordellii for pulsed-field electrophoresis analysis was conducted at $37{ }^{\circ} \mathrm{C}$ in an atmosphere of $10 \% \mathrm{H}_{2}, 10 \% \mathrm{CO}_{2}$ and $80 \% \mathrm{~N}_{2}$ in an anaerobic chamber (Coy Laboratory Products, Inc). C. sordellii was grown on Nutrient Agar (25 g/L Nutrient Broth No. 2 (Oxoid), $3 \mathrm{~g} / \mathrm{L}$ yeast extract, $1 \mathrm{~g} / \mathrm{L}$ sodium thioglycolate, $15 \mathrm{~g} / \mathrm{L}$ agar, $0.375 \%$ glucose) to obtain single colonies prior to growth overnight in $20 \mathrm{~mL}$ BHI broth $(35 \mathrm{~g} / \mathrm{L}$ Bacto Brain Heart Infusion (BD), $1 \mathrm{~g} / \mathrm{L}$ sodium thioglycollate, $0.375 \%$ glucose). Purified C. sordellii genomic DNA was extracted from $5 \mathrm{~mL}$ of overnight BHI culture as previously described [59] with the omission of RNAse upon resuspension of DNA. To produce agarose plugs containing $C$. sordellii genomic DNA, overnight BHI cultures were used to inoculate fresh $20 \mathrm{ml}$ BHI broths to an $\mathrm{OD}_{600}$ of 0.1 . These broth cultures were then grown to an $\mathrm{OD}_{600}$ of $\sim 1.0$. The bacterial cells were pelleted from $10 \mathrm{ml}$ of culture at $2466 \mathrm{x} \mathrm{g}$ for $8 \mathrm{~min}$. Bacterial cells were washed in $10 \mathrm{ml}$ PIV buffer $(10 \mathrm{mM}$ Tris- $\mathrm{HCl}, 1 \mathrm{M} \mathrm{NaCl}, \mathrm{pH} 7.6)$ and pelleted at $2466 \times \mathrm{g}$ for $8 \mathrm{~min}$. Cells were resuspended in $1 \mathrm{ml}$ PIV buffer and mixed with an equal volume of $1.5 \%$ SeaPlaque Agarose (Lonza) in $0.5 \mathrm{x}$ TBE buffer (the agarose having been equilibrated to $50{ }^{\circ} \mathrm{C}$ ). The cell-containing agarose plugs were cast in $1.5 \mathrm{~mm}$-thick moulds (Bio-Rad) at room temperature (RT). The plugs were removed from the moulds and incubated at $37{ }^{\circ} \mathrm{C}$ for $2 \mathrm{~h}$ with gentle shaking in lysis buffer (0.5 M EDTA [pH 8], 0.5 \% Sarkosyl, $2.5 \mathrm{mg} / \mathrm{ml}$ lysozyme, $2 \mathrm{mg} / \mathrm{ml}$ deoxycholic acid). The plugs were then incubated in proteinase $\mathrm{K}$ buffer $(0.5 \mathrm{M}$ EDTA [pH 8], $0.5 \%$ Sarkosyl, $1 \mathrm{mg} / \mathrm{ml}$ proteinase K) at $50{ }^{\circ} \mathrm{C}$ overnight. Plugs were then washed $4 \times 15 \mathrm{~min}$ in $0.1 \mathrm{x}$ TE, followed by washing in $1 \mathrm{x} \mathrm{TE}$, all washing occurring at RT with gentle shaking. Plugs were stored in fresh $1 \mathrm{x}$ TE at $4{ }^{\circ} \mathrm{C}$. To linearise pCS1-type plasmids so as to ensure more accurate size comparisons, agarose plugs used in this experiment were incubated with or without restriction enzyme BlpI (New England Biolabs) in $450 \mu \mathrm{l}$ NEBuffer 4 (New England Biolabs). PFGE of both extracted genomic DNA and agarose gel plugs containing genomic DNA were performed using a $1 \%$ Pulsed Field Certified Agarose (Bio-Rad) gel in $0.5 \mathrm{x}$ TBE buffer using the CHEF-DR III System (Bio-Rad) at $15{ }^{\circ} \mathrm{C}$. A Mid-Range I PFG Marker (New England Biolabs) was used as a molecular size standard. Electrophoresis parameters were as follows: $6 \mathrm{~V} / \mathrm{cm}$, ramping pulse from 1 to $25 \mathrm{~s}$ for $25 \mathrm{~h}$. Gels were stained in GelRed (Biotium) and photographed under UV light.

\section{Southern Hybridisation}

DNA from pulsed-field gels was transferred to a nylon membrane (Roche) as previously described [60] with the following modifications: $2 \times 20 \mathrm{~min}$ incubations in 
denaturation solution, $2 \times 20 \mathrm{~min}$ incubations in neutralisation solution and a $48 \mathrm{~h}$ transfer. The blots were hybridised to a $t c s L$-specific PCR product amplified using the primers DLP236 and DLP237, a pCS1 parBspecific PCR product amplified using the primers DLP377 and DLP378 or a ATCC9714PCS2_00141-specific PCR product amplified using the primers DLP362 and DLP363. All probes were amplified from C. sordellii ATCC9714 genomic DNA. Probes were digoxigeninlabelled using random PCR labelling according to the manufacturer's instructions (Roche). Hybridisation was detected using the CDP-Star (Roche) chemiluminescence detection system according to the manufacturer's instructions.

\section{Availability of supporting data}

The sequences described here have been deposited in the NCBI/EBI databases. Accession numbers for the genome of each strain are given in Table 1 . These are accessible through the WTSI website (http://www.sanger. ac.uk/resources/downloads/bacteria).

\section{Additional files}

Additional file 1: Putative Secreted Proteins of C. sordellii strain ATCC9714. Herein is contained a list of all putative secreted proteins encoded by the genome of C. sordellii ATCC9714, as identified by SignalP and Phobius, identifying them by gene number and indicating if they are proposed to be lipoproteins, if they are annotated with a specific gene product and giving a putative function based on BLAST analysis.

Additional file 2: Primers Used in This Study. Herein is contained a list of all primers used in this study, giving their name/number, sequence and what they were used for.

\section{Abbreviations}

LCC: Large clostridial cytotoxin; PFGE: Pulse field gel electrophoresis.

\section{Competing interests}

The authors declare they have no competing interests.

\section{Authors' contributions}

NF conceived the study. NF and TDL designed the study. EC, HPB and MD assembled and annotated the genome sequences and performed bioinformatics analyses. JGS, VH, LP and MA supplied C. sordellii strains and/ or genomic DNA and provided valuable insight into C. sordellii biology. CV performed Southern blot analysis; EC and NF drafted the manuscript and EC, $N F, D L, M A, C V, J G S$, and HP contributed to preparation of the final manuscript. All authors read and approved the final manuscript.

\section{Acknowledgments}

We thank Nick Thomson for advice on phylogenetics analysis. E.C. was funded by a BBSRC studentship. N.F. acknowledges a Research Fellowship from the Leverhulme Trust. This work was also supported by the Australian National Health and Medical Research Council (NHMRC). DL was supported by ARC Future Fellowship FT120100779 from the Australian Research

Council. T.L was funded by the Wellcome Trust grants 098051 and 086418.

\section{Author details}

'Department of Life Sciences, Centre for Molecular Bacteriology and Infection, Imperial College London, London SW7 2AZ, UK. 'Wellcome Trust Sanger Institute, Hinxton, UK. ${ }^{3}$ Department of Veterinary Science and Microbiology, University of Arizona, Tucson, USA. ${ }^{4}$ Anaerobe Reference
Laboratory, University Hospital of Wales, Cardiff, UK. ${ }^{5}$ Veterinary Laboratories Agency, Addlestone, Surrey, UK. ${ }^{6}$ Department of Microbiology, Monash University, Clayton, VIC 3800, Australia.

Received: 18 September 2014 Accepted: 5 May 2015

Published online: 16 May 2015

\section{References}

1. Hall IC, Scott JP. Bacillus sordellii; a cause of malignant edema in man. J Infect Dis. 1927;41:329-35.

2. Ryan J, Murphy C, Twomey C, Paul Ross R, Rea MC, MacSharry J, et al. Asymptomatic carriage of Clostridium difficile in an Irish continuing care institution for the elderly: prevalence and characteristics. Ir J Med Sci. 2010;179(2):245-50.

3. Aldape MJ, Bryant AE, Stevens DL. Clostridium sordellii infection: epidemiology, clinical findings, and current perspectives on diagnosis and treatment. Clin Infect Dis. 2006;43(11):1436-46.

4. File TM, Fass RJ, Perkins RL. Pneumonia and empyema caused by Clostridium sordellii. Am J Med Sci. 1977:274(2):211-2.

5. Gredlein CM, Silverman ML, Downey MS. Polymicrobial septic arthritis due to Clostridium species: case report and review. Clin Infect Dis. 2000;30(3):590-4.

6. Fischer M, Bhatnagar J, Guarner J, Reagan S, Hacker JK, Van Meter SH, et al. Fatal toxic shock syndrome associated with Clostridium sordellii after medical abortion. N Engl J Med. 2005;353(22):2352-60.

7. Aronoff DM, Hao Y, Chung J, Coleman N, Lewis C, Peres CM, et al. Misoprostol impairs female reproductive tract innate immunity against Clostridium sordellii. J Immunol. 2008;180(12):8222-30.

8. Lewis CJ, Naylor RD. Sudden death in sheep associated with Clostridium sordellii. Vet Rec. 1998;142(16):417-21.

9. Unger-Torroledo L, Straub R, Lehmann AD, Graber F, Stahl C, Frey J, et al. Lethal toxin of Clostridium sordellii is associated with fatal equine atypical myopathy. Vet Microbiol. 2010;144(3-4):487-92.

10. Aronoff DM. Clostridium novyi, sordellii, and tetani: Mechanisms of disease. Anaerobe. 2013;24:98-101.

11. Just I, Gerhard R. Large clostridial cytotoxins. Rev Physiol Biochem Pharmacol. 2004;152(1):23-47.

12. Martinez RD, Wilkins TD. Comparison of Clostridium sordellii toxins HT and LT with toxins A and B of C. difficile. J Med Microbiol. 1992;36(1):30-6.

13. Carter GP, Awad MM, Hao Y, Thelen T, Bergin IL, Howarth PM, et al. TcsL is an essential virulence factor in Clostridium sordellii ATCC 9714. Infect Immun. 2011;79(3):1025-32.

14. Walk ST, Jain R, Trivedi I, Grossman S, Newton DW, Thelen T, et al. Non-toxigenic Clostridium sordellii: clinical and microbiological features of a case of cholangitis-associated bacteremia. Anaerobe. 2011;17(5):252-6.

15. Hao Y, Senn T, Opp JS, Young VB, Thiele T, Srinivas G, et al. Lethal toxin is a critical determinant of rapid mortality in rodent models of Clostridium sordellii endometritis. Anaerobe. 2010;16(2):155-60.

16. Voth DE, Martinez OV, Ballard JD. Variations in lethal toxin and cholesteroldependent cytolysin production correspond to differences in cytotoxicity among strains of Clostridium sordellii. FEMS Microbiol Lett. 2006;259(2):295-302.

17. Sirigi Reddy AR, Girinathan BP, Zapotocny R, Govind R. Identification and characterization of Clostridium sordellii toxin gene regulator. J Bacteriol. 2013;195(18):4246-54.

18. Dupuy B, Govind R, Antunes A, Matamouros S. Clostridium difficile toxin synthesis is negatively regulated by TcdC. J Med Microbiol. 2008;57(Pt 6):685-9.

19. Mesnage S, Fontaine T, Mignot T, Delepierre M, Mock M, Fouet A. Bacterial SLH domain proteins are non-covalently anchored to the cell surface via a conserved mechanism involving wall polysaccharide pyruvylation. EMBO J. 2000;19(17):4473-84.

20. Tweten RK. Cholesterol-dependent cytolysins, a family of versatile pore-forming toxins. Infect Immun. 2005;73(10):6199-209.

21. Soltani CE, Hotze EM, Johnson AE, Tweten RK. Structural elements of the cholesterol-dependent cytolysins that are responsible for their cholesterolsensitive membrane interactions. Proc Natl Acad Sci U S A. 2007;104(51):20226-31.

22. Chien CH, Shann YJ, Sheu SY. Site-directed mutations of the catalytic and conserved amino acids of the neuraminidase gene, $n a n H$, of Clostridium perfringens ATCC 10543. Enzyme Microb Technol. 1996;19(4):267-76. 
23. Aldape MJ, Bryant AE, Ma Y, Stevens DL. The leukemoid reaction in Clostridium sordellii infection: neuraminidase induction of promyelocytic cell proliferation. J Infect Dis. 2007;195(12):1838-45

24. Karasawa T, Wang X, Maegawa T, Michiwa Y, Kita H, Miwa K, et al. Clostridium sordellii phospholipase C: gene cloning and comparison of enzymatic and biological activities with those of Clostridium perfringens and Clostridium bifermentans phospholipase C. Infect Immun. 2003;71(2):641-6.

25. Naylor CE, Eaton JT, Howells A, Justin N, Moss DS, Titball RW, et al. Structure of the key toxin in gas gangrene. Nat Struct Biol. 1998;5(8):738-46.

26. Eckhard U, Schönauer E, Nüss D, Brandstetter H. Structure of collagenase G reveals a chew-and-digest mechanism of bacterial collagenolysis. Nat Struct Mol Biol. 2011;18(10):1109-14.

27. Laarman AJ, Ruyken M, Malone CL, van Strijp JA, Horswill AR, Rooijakkers SH. Staphylococcus aureus metalloprotease aureolysin cleaves complement C3 to mediate immune evasion. J Immunol. 2011;186(11):6445-53.

28. Sieprawska-Lupa M, Mydel P, Krawczyk K, Wójcik K, Puklo M, Lupa B, et al. Degradation of human antimicrobial peptide LL-37 by Staphylococcus aureusderived proteinases. Antimicrob Agents Chemother. 2004;48(12):4673-9.

29. von Pawel-Rammingen U, Johansson BP, Björck L. IdeS, a novel streptococcal cysteine proteinase with unique specificity for immunoglobulin G. EMBO J. 2002:21(7):1607-15.

30. Fu HL, Valiathan RR, Arkwright R, Sohail A, Mihai C, Kumarasiri M, et al. Discoidin domain receptors: unique receptor tyrosine kinases in collagenmediated signaling. J Biol Chem. 2013;288(11):7430-7.

31. Burrows LL. Pseudomonas aeruginosa twitching motility: type IV pili in action. Annu Rev Microbiol. 2012;66:493-520.

32. Tataki $\mathrm{H}$, Huet M. Value of urease test for differentiation of Clostridium sordellii and Clostridium bifermentans. Ann Inst Pasteur (Paris). 1953:84(5):890-4.

33. Nakamura $\mathrm{S}$, Shimamura T, Nishida S. Urease-negative strains of Clostridium sordellii. Can J Microbiol. 1976;22(5):673-6.

34. Rutherford JC. The emerging role of urease as a general microbial virulence factor. PLoS Pathog. 2014;10(5), e1004062.

35. Dupuy B, Daube G, Popoff MR, Cole ST. Clostridium perfringens urease genes are plasmid borne. Infect Immun. 1997;65(6):2313-20.

36. Benini S, Rypniewski WR, Wilson KS, Miletti S, Ciurli S, Mangani S. A new proposal for urease mechanism based on the crystal structures of the native and inhibited enzyme from Bacillus pasteurii: why urea hydrolysis costs two nickels. Structure. 1999;7(2):205-16.

37. Paredes CJ, Alsaker KV, Papoutsakis ET. A comparative genomic view of clostridial sporulation and physiology. Nat Rev Microbiol. 2005;3(12):969-78.

38. Hoch JA. Regulation of the phosphorelay and the initiation of sporulation in Bacillus subtilis. Annu Rev Microbiol. 1993:47:441-65.

39. Underwood S, Guan S, Vijayasubhash V, Baines SD, Graham L, Lewis RJ, et al. Characterization of the sporulation initiation pathway of Clostridium difficile and its role in toxin production. J Bacteriol. 2009;191(23):7296-305.

40. Permpoonpattana P, Phetcharaburanin J, Mikelsone A, Dembek M, Tan S, Brisson MC, et al. Functional characterization of Clostridium difficile spore coat proteins. J Bacteriol. 2013;195(7):1492-503.

41. Sylvestre P, Couture-Tosi E, Mock M. A collagen-like surface glycoprotein is a structural component of the Bacillus anthracis exosporium. Mol Microbiol. 2002;45(1):169-78.

42. Liggins M, Ramirez N, Magnuson N, Abel-Santos E. Progesterone analogs influence germination of Clostridium sordellii and Clostridium difficile spores in vitro. J Bacteriol. 2011;193(11):2776-83.

43. Francis MB, Allen CA, Shrestha R, Sorg JA. Bile acid recognition by the Clostridium difficile germinant receptor, $\mathrm{CspC}$, is important for establishing infection. PLoS Pathog. 2013;9(5), e1003356.

44. Thiele TL, Stuber TP, Hauer PJ. Detection of Clostridium sordellii strains expressing hemorrhagic toxin (TcsH) and implications for diagnostics and regulation of veterinary vaccines. Vaccine. 2013;31(44):5082-7.

45. Bouvet P, Sautereau J, Le Coustumier A, Mory F, Bouchier C, Popoff MR. Foot Infection by Clostridium sordellii: Case Report and Review of 15 Cases in France. J Clin Microbiol. 2015;53(4):1423-7.

46. Abdulla A, Yee $L$. The clinical spectrum of Clostridium sordellii bacteraemia: two case reports and a review of the literature. J Clin Pathol. 2000;53(9):709-12.

47. Valour F, Boisset S, Lebras L, Martha B, Boibieux A, Perpoint T, et al. Clostridium sordellii brain abscess diagnosed by $16 \mathrm{~S}$ rRNA gene sequencing. J Clin Microbiol. 2010;48(9):3443-4.
48. Drudy D, Fanning S, Kyne L. Toxin A-negative, toxin B-positive Clostridium difficile. Int J Infect Dis. 2007;11(1):5-10.

49. Collins DA, Hawkey PM, Riley TV. Epidemiology of Clostridium difficile infection in Asia. Antimicrob Resist Infect Contr. 2013:2(1):21.

50. Brouwer MS, Roberts AP, Hussain H, Williams RJ, Allan E, Mullany P. Horizontal gene transfer converts non-toxigenic Clostridium difficile strains into toxin producers. Nat Commun. 2013;4:2601.

51. Schwartz DC, Li X, Hernandez LI, Ramnarain SP, Huff EJ, Wang YK. Ordered restriction maps of Saccharomyces cerevisiae chromosomes constructed by optical mapping. Science. 1993;262(5130):110-4.

52. Genome BiolBoetzer M, Pirovano W. Toward almost closed genomes with GapFiller. Genome Biol. 2012;13(6):R56.

53. Hyatt D, Chen GL, Locascio PF, Land ML, Larimer FW, Hauser LJ. Prodigal: prokaryotic gene recognition and translation initiation site identification. BMC Bioinformatics. 2010;11:119.

54. Seemann T. Prokka: rapid prokaryotic genome annotation. Bioinformatics. 2014;30(14):2068-9.

55. Fu L, Niu B, Zhu Z, Wu S, Li W. CD-HIT: accelerated for clustering the nextgeneration sequencing data. Bioinformatics. 2012;28(23):3150-2.

56. Edgar RC. MUSCLE: multiple sequence alignment with high accuracy and high throughput. Nucl Acids Res. 2004;32(5):1792-7.

57. Price MN, Dehal PS, Arkin AP. FastTree 2 - approximately maximum-likelihood trees for large alignments. PLos One. 2010;5(3), e9490.

58. Griffiths D, Fawley W, Kachrimanidou M, Bowden R, Crook DW, Fung R, et al. Multilocus sequence typing of Clostridium difficile. J Clin Microbiol. 2010;48(3):770-8.

59. O'Connor JR, Lyras D, Farrow KA, Adams V, Powell DR, Hinds J, et al. Construction and analysis of chromosomal Clostridium difficile mutants. Mol Microbiol. 2006;61(5):1335-51.

60. Sambrook J, Fritsch EF, Maniatis T. Molecular cloning. Cold Spring Harbor, New York: Cold Spring Harbor Press; 1989.

\section{Submit your next manuscript to BioMed Central and take full advantage of:}

- Convenient online submission

- Thorough peer review

- No space constraints or color figure charges

- Immediate publication on acceptance

- Inclusion in PubMed, CAS, Scopus and Google Scholar

- Research which is freely available for redistribution 